\title{
EXPANSION OF THE ROTATIONAL ENERGY OF DIATOMIC MOLECULES INTO A CONTINUED FRACTION*
}

\author{
M. Molski and J. KonarsKi \\ Department of Theoretical Chemistry, Faculty of Chemistry, A. Mickiewicz University \\ Grunwaldzka 6, 60-780 Poznań, Poland
}

(Received January 21, 1992; in final form March 30, 1992)

\begin{abstract}
A new expansion of the rotational energy of diatomic molecules, in the form of a continued fraction has been investigated. The considered formula is applied in calculation of molecular constants and reproduction of rotational spectra of rigid-, semirigid- and van der Waals-type molecules. A physical interpretation of semiempirical expansion parameters is proposed and a connection between the method considered and the Dunham approach is also discussed.
\end{abstract}

PACS numbers: $33.10 . \mathrm{Cs}, 33.10 . \mathrm{Jz}$

\section{Introduction}

A simple analysis of molecular rotational-vibrational (rovibrational) spectra has been realized in the framework of the potential approach as well as the Dunham method. In the potential approach, energy levels are obtained by solving the Schrödinger equation with the interatomic potential expanded in terms of interatomic variables [1-4]. The potential coefficients are obtained either by a fit to the experimental energy levels or, if available, by a fit to a theoretically calculated potential. In the above method the solution of the Schrödinger equation provides wave functions which can be applied in evaluation of matrix elements of various quantum-mechanical operators essential in a more sophisticated analysis of molecular spectra.

The second popular method of assignment of IR and MW spectra is the Dunham approach [5] where the rovibrational energy is expanded in terms of vibrational and rotational quantum numbers $v$ and $J$, respectively. For diatomic molecules this yields

$$
E_{v J}=\sum_{k, l} Y_{k l}(v+1 / 2)^{k} J^{l}(J+1)^{l}
$$

\footnotetext{
*This research was supported by KBN grant 2066391.01 .
} 
where $Y_{k l}$ are semiempirical parameters whose values are obtained by a fit to the experimental energy levels. This method has been applied to many molecules, giving quite satisfactory relations between theoretical and experimental data [6], as well as providing information on interatomic molecular potential $[7,8]$. The disadvantage of the Dunham approach is that a power series describing rovibrational energy has a poor convergence, and that Dunham expansion does not provide any information about the wave function of individual states of a molecule. In order to avoid the above difficulties, more advanced and adequate methods based on different approaches [9-16] have been suggested.

In view of the above consideration, the main purpose of this work is to present a new method which combines the potential and the Dunham approach, and may be used in an analysis of the rotational spectra of diatomic molecules. The considered formula will be applied in evaluation of molecular constants and in reproduction of the energy of rotational transitions of the ${ }^{1} \Sigma$ state of ${ }^{12} \mathrm{C}^{16} \mathrm{O}$, $\mathrm{H}^{35} \mathrm{Cl}$ and of the ${ }^{1} \Sigma_{g}$ state of ${ }^{40} \mathrm{Ar}_{2}$ molecules.

\section{General formulation}

Let us consider a two-atom molecule endowed with a reduced mass $m=$ $m_{1} m_{2} /\left(m_{1}+m_{2}\right)$ where $m_{1}$ and $m_{2}$ are masses of the molecule constituents. If we assume that molecular potential has a simple Simons-Parr-Finlan form [2, 3] the rotation-vibration wave equation for such a system may be written as follows:

$$
\left[-\frac{\hbar^{2}}{2 m} \frac{\partial^{2}}{\partial q^{2}}+\frac{\hbar^{2} J(J+1)}{2 m\left(q_{0}+q\right)^{2}}+D_{0}\left(1-\frac{q_{0}}{q}\right)^{2}-E_{v J}\right] \psi_{v J}=0
$$

where $q$ describes the distance between the two atoms, $q_{0}$ is an equilibrium distance and $D_{0}$ is a constant related to the dissociation energy of the molecule. Taking into account a quantum-mechanical equation of motion in the Heisenberg representation, as well as the explicit form of Hamilton's operator occurring in (2), we get

$$
\begin{aligned}
& \hat{\dot{p}}=(\mathrm{i} \hbar)^{-1}[\hat{p}, \hat{H}]=-\frac{2 D_{J} q_{J}\left(q-q_{J}\right)}{q^{3}}, \\
& D_{J}=D_{0}\left[1+B_{0} D_{0}^{-1} J(J+1)\right]^{-1}, \quad B_{0}=\hbar^{2}\left(2 m q_{0}^{2}\right)^{-1}, \\
& q_{J}=q_{0}\left[1+B_{0} D_{0}^{-1} J(J+1)\right] .
\end{aligned}
$$

The right hand side of (3) represents the quantum-mechanical force which has a potential

$$
U_{\text {ef }}=D_{J}\left(1-\frac{q_{J}}{q}\right)^{2}
$$

whereas the quantities $D_{J}$ and $q_{J}$ are the modified dissociation constant and the changed equilibrium distance, respectively.

A look into (3) reveals that in rovibrational two-atomic systems, described by the Simons-Parr-Finlan potential, a time-change of momentum is a result of force 
with the modified dissociation constant $D_{J}$, and the changed equilibrium distance $q_{J}$. This is a simple consequence of the deformational action of the centrifugal force which acts upon systems with rotational degree of freedom [17]. In view of the above, the Schrödinger equation (2) may be rewritten into the equivalent formula

$$
\left[-\frac{\hbar^{2}}{2 m} \frac{\partial^{2}}{\partial q^{2}}+D_{J}\left(1-\frac{q_{J}}{q}\right)^{2}+\frac{B_{0} J(J+1)}{1+B_{0} D_{0}^{-1} J(J+1)}-E_{v J}\right] \psi_{v J}=0
$$

which can be strictly solved $[18,19]$, leading to the energy eigenvalues and the corresponding wave functions given in the forms

$$
\begin{aligned}
& E_{v J}=D_{J}\left\{1-\frac{4 D_{J} B_{J}^{-1}}{\left[1+2 v+\left(1+4 D_{J} B_{J}^{-1}\right)^{1 / 2}\right]^{2}}\right\}+\frac{B_{0} J(J+1)}{1+B_{0} D_{0}^{-1} J(J+1)} \\
& B_{J}=\hbar^{2}\left(2 m q_{J}^{2}\right)^{-1}=B_{0}\left[1+B_{0} D_{0}^{-1} J(J+1)\right]^{-2} \\
& \psi_{v J}=N_{v J}\left(2 \beta_{v J} \rho\right)^{\alpha_{J}} \exp \left(-\beta_{v J} \rho\right) L_{v}^{\gamma_{J}}\left(2 \beta_{v J} \rho\right), \\
& L_{v}^{\gamma_{J}}\left(2 \beta_{v J} \rho\right)=\frac{\Gamma\left(1+\gamma_{J}+v\right)}{v ! \Gamma\left(1+\gamma_{J}\right)}{ }_{1} F_{1}\left(-v ; 1+\gamma_{J} ; 2 \beta_{v J} \rho\right), \\
& N_{v J}=\left[\frac{v !}{2\left(\gamma_{J}+v\right) \Gamma\left(1+\gamma_{J}+v\right)}\right]^{1 / 2}
\end{aligned}
$$

where $L_{v}^{\gamma_{J}}\left(2 \beta_{v J} \rho\right)$ is the generalized Laguerre polynomial, ${ }_{1} F_{1}\left(-v ; 1+\gamma_{J} ; 2 \beta_{v J} \rho\right)$ denotes the confluent hypergeometric (or Kummer) function whereas

$$
\begin{aligned}
& \rho=\frac{q}{q_{J}}, \quad \alpha_{J}=\frac{1+\gamma_{J}}{2}, \\
& \gamma_{J}=\sqrt{1+4 D_{J} B_{J}^{-1}}, \quad \beta_{v J}=\frac{D_{J}}{B_{J}\left(v+\alpha_{J}\right)} .
\end{aligned}
$$

The obtained results indicate that rovibrational energy of diatomic systems consists of an effective vibration energy described by the first term in (7), and an effective rotation energy given by the second one. It is easy to verify that

$$
E_{\mathrm{ef}}^{\mathrm{Rot}}=\frac{B_{0} J(J+1)}{1+B_{0} D_{0}^{-1} J(J+1)}=D_{0}\left(1-\frac{q_{0}}{q_{J}}\right)^{2}+B_{J} J(J+1)
$$

i.e., the effective rotational energy is a result of a change of the equilibrium configuration $q_{0} \rightarrow q_{J}$ caused by the centrifugal force.

\section{Applications}

The formula (7) may be applied in calculation of the rovibrational energy of diatomic molecules, however, in the case of rotational transitions for low excited vibrational states $(v=0,1)$, in the first approximation one may neglect rotational 
dependence of the effective vibrational energy. As a result, the simplified energy formula for rotational transitions reads

$$
\Delta E_{J}=\frac{B_{0}(J+1)(J+2)}{1+B_{0} D_{0}^{-1}(J+1)(J+2)}-\frac{B_{0} J(J+1)}{1+B_{0} D_{0}^{-1} J(J+1)} .
$$

In the zero order approximation considering the molecule as a rigid rotor $\left(D_{0}=\infty\right)$, Eq. (12) reduces to the well-known formula $\Delta E_{J}=2 B_{0}(J+1)$.

A detailed analysis of (11) and (12) leads to the following conclusions:

(i) For a small value of the constant $B_{0} D_{0}^{-1}$, the effective rotational energy can be expanded in a power series of $J(J+1)$, leading to the polynomial Dunham expansion of the rotational energy of diatomic molecules. So, the Dunham formula is a particular case of the general one given by the analytical equation (7) or the approximated expression (11).

(ii) The continued fraction formula (11) is a more general and stronger physically supported equation describing the rotational energy of two-atom systems, then the Dunham proposal.

In view of the above, it is tempting to employ in reproduction of the energy of rotational transitions, a polyparametric continued fraction formula

$$
E_{J}=\frac{B_{0} J(J+1)}{1+\frac{B_{1} J(J+1)}{1+\frac{B_{2} J(J+1)}{1+\cdots \ldots . .}}},
$$

and compare the calculated frequencies with these obtained by making use of Eq. (1). The molecules ${ }^{12} \mathrm{C}^{16} \mathrm{O}, \mathrm{H}^{35} \mathrm{Cl}$, and ${ }^{40} \mathrm{Ar}_{2}$ will be taken as an example.

The best values of the molecular parameters will be determined by the linear least-square routine in which the statistical weights, proportional to the inverse of experimental uncertainties, are taken as being equal to one. In Table I the parameters $\left\{B_{k}, k=0,1,2 \ldots\right\}$ calculated on the basis of the $N$-parametric continued fraction formula (13) are tabulated, whereas Table II presents the Dunham parameters $\left\{Y_{0 J}, J=0,1,2 \ldots.\right\}$ obtained by using the $N$-parametric polynomial expansion

$$
\begin{aligned}
E_{J}= & B J(J+1)-D J^{2}(J+1)^{2}+H J^{3}(J+1)^{3}-L J^{4}(J+1)^{4} \\
& +M J^{5}(J+1)^{5}-\ldots \ldots .
\end{aligned}
$$

\section{Discussion}

The performed calculations lead to the conclusion that the equation better physically supported and describing correctly the effective rotation energy of the considered molecules, is the 3-parametric continued fraction

$$
E_{\mathrm{ef}}^{\mathrm{Rot}}=\frac{B_{0} J(J+1)}{1+\frac{B_{0_{0} J(J+1)}}{D_{0}\left[1+B_{2} J(J+1)\right]}},
$$

to be obtained from (11) by a formal substitution

$$
D_{0} \rightarrow D(J)=D_{0}\left[1+B_{2} J(J+1)\right] \text {. }
$$


This indicates that the dissociation constant depends on the rotational quantum number $J$. Because the semiempirical parameter $B_{2}$ is negative for all the cases considered, it becomes apparent that the dissociation energy of rotating systems diminishes according to formula (16). This effect, for highly excited rotational states, leads to the possibility of rotation induced dissociation of a molecule. Therefore, the semiempirical parameter $B_{2}$ may be viewed as an indicator of molecular susceptibility to rotational dissociation, which, according to our intuitive expectations, diminshes with rigidity of a molecule (see Table I). The remaining parameters

Continued fraction molecular parameters ${ }^{a} B_{k}$ fitted to the experimental data.

TABLE I

\begin{tabular}{l|c|c|c|c|c}
\hline \hline & $\sigma_{N}^{b}$ & $B_{0}$ & $B_{1}$ & $B_{2}$ & $B_{3}$ \\
\hline $\mathrm{MHz}$ & 5.125 & $57636.44(11)$ & $3.1992(11) \cdot 10^{-6}$ & & \\
${ }^{12} \mathrm{C}^{16} \mathrm{O}$ & 0.051 & $57635.9649(18)$ & $3.183843(49) \cdot 10^{-6}$ & $-2.2423(70) \cdot 10^{-6}$ & \\
$8^{c}$ & 0.050 & $57635.9667(24)$ & $3.18399(14) \cdot 10^{-6}$ & $-2.177(56) \cdot 10^{-6}$ & $-1.1(10) \cdot 10^{-5}$ \\
\hline $\mathrm{MHz}$ & 9.599 & $312991.80(62)$ & $5.0756(14) \cdot 10^{-6}$ & & \\
$\mathrm{H}^{35} \mathrm{Cl}$ & 0.077 & $312989.2446(79)$ & $5.057881(46) \cdot 10^{-6}$ & $-1.924(9) \cdot 10^{-5}$ & \\
10 & 0.071 & $312298.9253(12)$ & $5.05800(13) \cdot 10^{-6}$ & $-1.894(34) \cdot 10^{-5}$ & $-6.9(78) \cdot 10^{-5}$ \\
\hline $\mathrm{Cm}^{-1}$ & 0.078 & $5.8115(53) \cdot 10^{-2}$ & $2.553(53) \cdot 10^{-5}$ & & \\
${ }^{40} \mathrm{Ar}_{2}$ & 0.008 & $5.76854(93) \cdot 10^{-2}$ & $1.555(16) \cdot 10^{-5}$ & $-1.361(23) \cdot 10^{-4}$ & \\
25 & 0.006 & $5.7727(13) \cdot 10^{-2}$ & $1.708(39) \cdot 10^{-5}$ & $-8.2(11) \cdot 10^{-5}$ & $-1.17(26) \cdot 10^{-4}$ \\
\hline
\end{tabular}

${ }^{a}$ For ${ }^{12} \mathrm{C}^{16} \mathrm{O}, \mathrm{H}^{35} \mathrm{Cl}, \sigma_{N}$ and $B_{0}$ are calculated in $\mathrm{MHz}$, and for ${ }^{40} \mathrm{Ar}_{2}$ in $\mathrm{cm}^{-1}$. Remaining parameters are dimensionless.

b Standard deviation of the fit.

c Number of experimental data in the fit.

TABLE II

Durham molecular parameters ${ }^{a} Y_{0 J}$ fitted to the experimental data.

\begin{tabular}{l|c|c|c|c|c}
\hline \hline & $\sigma_{N}^{b}$ & $B$ & $D$ & $H$ & $L$ \\
\hline $\mathrm{MHz}$ & 2.140 & $57635.762(45)$ & $0.183133(27)$ & & \\
${ }^{12} \mathrm{C}^{16} \mathrm{O}$ & 0.051 & $57635.9648(19)$ & $0.1835036(29)$ & $1.723(13) \cdot 10^{-7}$ & \\
$8^{c}$ & 0.050 & $57635.9667(24)$ & $0.1835128(83)$ & $1.848(105) \cdot 10^{-7}$ & $4.94(41) \cdot 10^{-12}$ \\
\hline $\mathrm{MHz}$ & 15.519 & $\mathbf{3 1 2 9 8 5 . 1 1 ( 9 9 )}$ & $15.7411(70)$ & & \\
$\mathrm{H}^{35} \mathrm{Cl}$ & 0.077 & $\mathbf{3 1 2 9 8 9 . 2 3 8 6 ( 8 8 )}$ & $15.83036(16)$ & $4.9240(87) \cdot 10^{-4}$ & \\
10 & 0.071 & $312298.9252(12)$ & $15.83098(42)$ & $5.009(55) \cdot 10^{-4}$ & $3.6(24) \cdot 10^{-8}$ \\
\hline $\mathrm{cm}^{-1}$ & 0.067 & $5.564(15) \cdot 10^{-2}$ & $1.377(24) \cdot 10^{-6}$ & & \\
${ }^{40} \mathrm{Ar}_{2}$ & 0.012 & $5.8048(43) \cdot 10^{-2}$ & $0.771(24) \cdot 10^{-6}$ & $-1.950(77) \cdot 10^{-10}$ & - \\
25 & 0.005 & $5.7758(15) \cdot 10^{-2}$ & $1.095(35) \cdot 10^{-6}$ & $5.3(26) \cdot 10^{-11}$ & $5.66(58) \cdot 10^{-14}$ \\
\hline
\end{tabular}

a For ${ }^{12} \mathrm{C}^{16} \mathrm{O}, \mathrm{H}^{35} \mathrm{Cl}$, Dunham parameters and $\sigma_{N}$ are calculated in $\mathrm{MHz}_{\text {, and for }}{ }^{40} \mathrm{Ar}_{2}$ in $\mathrm{cm}^{-1}$.

b Standard deviation of the fit.

c Number of experimental data in the fit.

in the 3-parametric expansion (15) have a clear physical interpretation, namely, $B_{0}=\hbar^{2}\left(2 m q_{0}^{2}\right)^{-1}, B_{1}=\hbar^{2}\left(2 m D_{0} q_{0}^{2}\right)^{-1}$ and may be used to determine the equilibrium distance $q_{0}$ and the dissociation constant $D_{0}$ of the two-atom systems under consideration. Additional results can be summarized as follows: 
(i) Starting from the well physically defined model of a rotating diatomic molecule it is possible to reproduce with high accuracy the observed transitions in a wide range of rotational states.

(ii) Application of the 4th semiempirical parameter practically does not give a remarkable change in the accuracy of calculations.

(iii) To the accuracy of $0.01 \mathrm{MHz}$ and $0.01 \mathrm{~cm}^{-1}$, quoted in Refs. [20] and [21], the energy of the rotational transitions reproduced by the 3-parametric continued fraction (13) is in perfect agreement with that provided by the 3-parametric Dunham expansion (14). Although, it is worth noting the difference at the 3rd decimal position points to the advantage of the former, both for rigid$\left({ }^{12} \mathrm{C}^{16} \mathrm{O}\right)$, semirigid- $\left(\mathrm{H}^{35} \mathrm{Cl}\right)$ and van der Waals $\left({ }^{40} \mathrm{Ar}_{2}\right)$-type of molecules.

(iv) From Table I we see that for ${ }^{40} \mathrm{Ar}_{2}$, application of the sextic parameter in the fit leads to very good theoretical reproduction of the experimental data and results in a value for the quartic constant which is an order of magnitude lower, so, we arrive at the conclusion that the $N$-parametric fit is an "effective" fit for the problem considered. It should be mentioned, moreover, that in Ref. [21] only a quartic fit to the lowest rotational states was reported.

(v) The Dunham expansion of the rotational energy of two-atom systems may be treated as a particular case of the general expression (13).

(vi) The presented approach allows the wave function to be easily obtained by the formal substitution $D_{0} \rightarrow D_{0}\left[1+B_{2} J(J+1)\right]$ in (9), so it is possible to avoid the main disadvantage of the Dunham approach.

The proposed method may also be applied to molecules for which the experimental data allows determination of octic and decadic constants, such as HF, by making use of the 4 - and 5-parametric continued fractions, respectively. The results of investigation of this and other molecules will be presented in a separate paper.

\section{Acknowledgment}

We are indebted to the referee for bringing our attention to the problem of the fit effectivity mentioned in discussion, as well as for referring us to the work of Nakagawa and Uehara*.

* Note added in proof: The ${ }^{40} \mathrm{Ar}_{2}$ and ${ }^{12} \mathrm{C}^{16} \mathrm{O}$ molecules considered in our paper have been also investigated by K. Nakagawa and H. Uehara in Chem. Phys. Lett. 168, 96 (1990), who applied however quite a different approach. Our calculations start with the supposition of rotational dependence of a dissociation constant (see Eqs. (15) and (16)), whereas in the paper mentioned the standard multiparametric Simons-Parr-Finlan potential has been applied which provided (in the variational approach) a very good reproduction of the rovibrational transitions using six parameters fitted to experimental data. The method presented in our paper concerns only rotational states of molecules, and is based on another set of fitted parameters $\left(B_{k}, k=0,1,2\right)$, which gives the same accuracy of the calculated results. 


\section{References}

[1] T.G. Waech, R.B. Berstein, J. Chem. Phys. 46, 4905 (1967).

[2] G. Simons, R.G. Parr, J.M. Finlan, J. Chem. Phys. 59, 3229 (1973).

[3] G. Simons, J.M. Finlan, Phys. Rev. Lett. 33, 131 (1974).

[4] J. Konarski, Teoretyczne Podstawy Spektroskopii Molekularnej, PWN, Warszawa 1991 (and references cited therein) (in Polish).

[5] J.L. Dunham, Phys. Rev. 41, 713 (1932).

[6] I.M. Mils, Specialist Periodical Report-Theoretical Chemistry, Vol. 1, Amer. Chem. Soc., Washington DC 1974, p. 110.

[7] R.N. Zare, A.L. Schmeltekopf, W.J. Harrod, D.L. Albritton, J. Mol. Spectrosc. 46, 37 (1973).

[8] D.L. Albritton, W.J. Harrod, A.L. Schmeltekopf, R.N. Zare, J. Mol. Spectrosc. 46, 67 (1973).

[9] A.J. Thakar, J. Chem. Phys. 62, 1693 (1975).

[10] N.S. Mohammad, Int. J. Quantum Chem. 17, 943 (1980).

[11] J.N. Huffaker, J. Chem. Phys. 64, 3175 (1976); 64, 4565 (1976).

[12] Y. Makushin, O.V. Naumienko, O.N. Ulenikow, J. Mol. Spetrosc. 103, 221 (1984).

[13] V.I. Pierevalov, V.G. Tyuterev, B.I. Zhilinski, J. Mol. Spetrosc. 103, 147 (1984).

[14] K.S. Jordan, J.L. Kinsey, R. Silbey, J. Chem. Phys. 61, 911 (1974).

[15] J. Konarski, J. Mol. Spetrosc. 124, 218 (1987).

[16] J. Konarski, Acta Phys. Pol. A74, 235 (1988).

[17] M. Molski, Acta Phys. Pol. A76, (1989).

[18] A. Kratzer, Z. Phys. 3, 289 (1920).

[19] E. Fues, Ann. Phys. (Paris) 80, 367 (1926); 81, 281 (1926).

[20] I.G. Nolt, J.V. Radostitz, G. DiLonardo, K.M. Evenson, D.A. Jennings, K.R. Leopold, M.D. Vanek, L.R. Zink, A. Hinz, K.V. Chance, J. Mol. Spetrosc. 125, 274 (1987).

[21] E.A. Colbourn, A.E. Douglas, J. Chem. Phys. 65, 1741 (1976). 